activation and inflammation in the genital tracts of adolescents from South Africa.

Methods Cervical cytobrush mononuclear cells were isolated from 148 adolescents (16-22 years) from Cape Town, and expression of T-cell activation and proliferation markers (CD38, HLA-DR, Ki67, CCR5) was measured by FACs. Adolescents were screened for BV (Nugent) and STIs (C. trachomatis, N. gonnorhoea, T. vaginalis, M. genitalium, HSV-2) by PCR. For comparison, 11 HIV-negative adult women were included. Concentrations of 48 cytokines, chemokines and growth factors were measured in matching menstrual cups by Luminex.

Results Adolescents (median 18 years; IQR 17-20) had significantly higher frequencies of activated $\mathrm{CD} 4^{+}$T-cells $\left(\mathrm{CD} 38^{+}\right.$, $\mathrm{HLADR}^{+}, \mathrm{CD}_{3} 8^{+} \mathrm{HLADR}^{+}$: each $\left.\mathrm{p}<0.0001\right)$ from cervical cytobrushes than adults although CCR5 expression was higher in adults. STIs and BV prevalence was very high, with $71 \%$ of adolescents having $\geq 1$ STI and/or BV, and $42 \%$ being C. trachomatis positive. Adolescents with an STI, despite these being asymptomatic, had higher frequencies of activated and proliferating T-cells compared to those with no STI/BV $\left(\mathrm{CD} 4{ }^{+} \mathrm{CD} 38^{+} \mathrm{HLADR}^{+}: \mathrm{p}=0.047 ; \mathrm{CD}^{+}{ }^{+} \mathrm{Ki}^{+}: \mathrm{p}=0.020\right)$. Women positive for chlamydia had significantly higher frequencies of $\mathrm{CD} 4{ }^{+} \mathrm{CD} 38^{+}$T-cells $(\mathrm{p}=0.006)$. Women with both STIs and BV had the most pronounced increase in $\mathrm{CD}^{+}{ }^{+} \mathrm{T}$-cell activation $\left(\mathrm{CD}^{+} 8^{+}: \mathrm{p}=0.002 ; \mathrm{CD}^{+} 8^{+} \mathrm{HLADR}^{+}: \mathrm{p}=0.001\right.$; Ki67+: $p=0.002)$. Higher cervical T-cell activation marker expression was directly associated with increased genital cytokine profiles.

Conclusion Heightened levels of genital immune activation and inflammation found in South African adolescent females, partly due to the presence of asymptomatic STIs and BV could increase their risk for HIV infection.

\section{P15.05 PERFORMANCE EVALUATION OF THE APTIMA HIV-1 QUANT DX ASSAY FOR DETECTION OF HIV-1 IN PLASMA AND DRIED BLOOD SPOTS (DBS)}

${ }^{1} \mathrm{~A}$ Carrera, ${ }^{1} \mathrm{~J}$ Sherring, ${ }^{1} \mathrm{~L}$ McNally, ${ }^{2} \mathrm{P}$ Lowe, ${ }^{1,3} \mathrm{PH}$ Cunningham*. ${ }^{1} \mathrm{NSW}$ State Reference Laboratory for HIV, St Vincent's Hospital Sydney Limited; ${ }^{2}$ Hologic Australia P/L; ${ }^{3}$ Kirby Institute UNSW

\subsection{6/sextrans-2015-052270.546}

Introduction The Aptima HIV-1 Quant Dx presented on the Hologic PANTHER ${ }^{\mathrm{TM}}$ system provides continuous and random access processing of molecular samples for groups M HIV-1 RNA viral load testing. Significant efficiencies are realised through $3.5 \mathrm{~h}$ to first result with over 275 samples processed within $8 \mathrm{~h}$. This study assessed the performance of the system in routine plasma samples and whole blood presented as dried blood spot (DBS).

Methods A total of 181 plasma samples were tested over the analytical range and compared to a benchmark real time PCR system. The study focused on the lower analytical range $<5,000$ copies/mL HIV-1 RNA (55\%). HIV-1 viral load equivalence in non-B subtypes of regional geographical significance was assessed where subtype was available (72\%). A further 20 DBS (single 10 mm punch, whole blood) with HIV-1 RNA 500-5,000 cpy/mL and were eluted using a variety of methods, tested and compared with plasma RNA.

Results Overall, Aptima HIV-1 Quant Dx correlated with the routine analytical platform $(\mathrm{r} 2=0.9605)$. Samples ranged undetectable $(16,8.8 \%)$, below the benchmark test lower limit of detection $(<20 \mathrm{cpy} / \mathrm{ml})(16,8.8 \%)$, low range $(20-5,000)(84$,
$46.4 \%)$, medium $(5,000-50,000)(36,19.9 \%)$ and high range $(>50,000 \mathrm{cpy} / \mathrm{ml})(29,16 \%)$. Samples in the lower analytical range $<1,000 \mathrm{cpy} / \mathrm{ml}$ showed little variance when compaired with the Roche (CAP/CTM) assay using Bland-Altman correlation analysis. Reproducibility was assessed in the high, medium and low range within 1-2SD of mean. DBS samples with HIV-1 RNA results $>1,000$ were well correlated with plasma.

Conclusion The Aptima HIV-1 Quant Dx automated random access platform correlated with a commonly used HIV RNA test in plasma and offered significant workflow advantages. Promising results obtained using DBS samples could potentially overcome logistics encountered with conventional plasma. Further correlations and limit of detection studies are needed to validated DBS.

Disclosure of interest statement No conflict of interest to declare.

\section{P15.06 MOLECULAR VALIDATION OF PUTATIVE ANTIMICROBIAL PEPTIDES FOR IMPROVED HUMAN IMMUNODEFICIENCY VIRUS DIAGNOSTICS VIA HIV PROTEIN P24}

${ }^{1} \mathrm{ME}$ Williams* ${ }^{*}{ }^{1} \mathrm{M}$ Tincho, ${ }^{1,2} \mathrm{M}$ Meyer, ${ }^{1,2} \mathrm{~A}$ Pretorius. ${ }^{1}$ University of the Western Cape; ${ }^{2}$ Nanotechnology Innovation Centre

\subsection{6/sextrans-2015-052270.547}

Introduction The Human Immunodeficiency Virus-1 (HIV-1) is responsible for causing Acquired Immunodeficiency Syndrome (AIDS), and to date remains a pandemic. More than 40 million people are infected globally, with $60 \%$ of the infected people residing in Sub-Saharan Africa. Earlier detection translates into earlier treatment, which ensures improved quality of life. However, difficulties remain in the field of HIV diagnostics. The p24 antigen detection tests are preferred due to its ability to decrease the window period. The current p24 diagnostic assay displays great insensitivity, due to the p24 antibody produced by the body, binding to the C-terminal of the p24 antigen. This interaction obstructs detection, the basis of the current p24 test. Using in silico approaches, novel antimicrobial peptides (AMP) were identified which bind to the N-terminal, instead of the C-terminal domain (antibody binding pocket) of the p24 antigen (provisional patent). This is important because if the p24 antibody binds to the C-terminal, the unoccupied $\mathrm{N}$-terminal domain would provide a binding pocket for the AMP. Successful conjugation of nanoparticles to the positively validated AMP, can lead to the development of a diagnostic lateral flow device.

Methods In silico site-directed mutagenesis and docking studies to identify additional AMPs that bind the N-terminal domain of protein $\mathrm{p} 24$ with increased binding affinity.

Preliminary study: Lateral flow design with identified AMPs to test HIV positive sera.

P24 recombinant protein expression.

P24 protein-AMP binding studies.

Results In silico studies identified 9 AMPs which could be used to bind p24 antigen for HIV diagnostics.

Preliminary study: Lateral flow successfully detected HIV in HIV positive sera.

Successful p24 recombinant protein expression.

Successful validation of binding AMPs against the p24 protein.

Conclusion Binding interaction between AMPs and p24 protein is validated. Subsequently a sensitive lateral flow device could be developed that successfully detects HIV in positive HIV sera. 
Disclosure of interest statement All research has been funded by the National Research Foundation and Nanotechnology Innovation Centre. No commercial funding has been provided for this study.

\section{P16 - HIV epidemiology}

\section{P16.01 AN AGENT-BASED MODEL TO SIMULATE AND PREDICT HIV EPIDEMIC IN BALTIMORE CITY, MARYLAND, USA}

1) Adleberg, ${ }^{1} \mathrm{R}$ Rothman, ${ }^{2} \mathrm{C}$ Flynn, ${ }^{3} \mathrm{C}$ Nganga-Good, ${ }^{1} \mathrm{Y}-\mathrm{H}$ Hsieh*. ${ }^{1}$ Department of Emergency Medicine, Johns Hopkins University School of Medicine, Baltimore, Maryland, USA; ${ }^{2}$ Maryland Department of Health and Mental Hygiene, Baltimore, Maryland, USA; ${ }^{3}$ Baltimore City Health Department, Baltimore, Maryland, USA

\subsection{6/sextrans-2015-052270.548}

Introduction Baltimore City has one of the highest HIV incidences and prevalences in the United States. An HIV testing program, implemented in several emergency departments (EDs), has accounted for $11 \%$ of newly diagnosed HIV cases from 20082013. We derive an agent-based model (ABM) for HIV transmission in Baltimore City, and use this to determine the significance of ED-based HIV testing on HIV transmission.

Methods An agent-based computational simulation was performed via the Python programming language, using 523,113 agents to represent the $13+$ population of Baltimore City. The simulation was calibrated using HIV prevalence and incidence data culled from 2007 to 2013 City surveillance data. During each timestep, agents interacted with other agents. Agents were assigned one of three categories: seronegative, seropositive aware, or seropositive unaware, and individual risks were assigned from these categories, with seropositive unaware agents being 3.5 times more likely to transmit the disease. ED testing changed unaware agents to aware, and rates of testing were varied in order to study the effects on overall incidence. A subsequent sensitivity analysis was performed using different ranges of parameters, and a range of incidence projections was calculated. Results Baltimore City HIV incidence decreased from 1,052 new cases $(0.207 \%)$ in 2007 to $356(0.068 \%)$ in 2013. Our model was able to approximate HIV incidence over time as observed from 2007 to 2013. Overall HIV incidence is forecast to decrease from $0.068 \%$ in 2013 to $0.042 \%$ in 2020 (95\% CI: $0.015-0.079$ ). It is further demonstrated that doubling capacity of the ED-based testing programs would likely avert 35 additional HIV transmissions from 2014 to 2020.

Conclusion We conclude that ABM provides an effective means of describing an epidemic with a highly heterogeneous population, and additionally that the ED-based testing program has had a significant impact on curtailing HIV transmission in Baltimore City.

Disclosure of interest statement This work was supported by a National Institutes of Health grant [K01AI100681 to Y-HH]. No conflicts of interest are reported.
P16.02 PREDICTORS OF HIV ACQUISITION WITHIN 12 MONTHS OF AN HIV NEGATIVE TEST IN MEN WHO HAVE SEX WITH MEN

${ }^{1,2} \mathrm{KT}$ Cheung ${ }^{*},{ }^{1,3} \mathrm{CK}$ Fairley, ${ }^{1,3} \mathrm{TRH}$ Read, ${ }^{1}$ I Denham, ${ }^{1} \mathrm{G}$ Fehler, ${ }^{1,3} \mathrm{CS}$ Bradshaw, ${ }^{1,3} \mathrm{MY}$ Chen, ${ }^{1,3} \mathrm{EPF}$ Chow. ${ }^{1}$ Melbourne Sexual Health Centre, Alfred Health, Melbourne, VIC Australia; ${ }^{2}$ Melbourne Medical School, Faculty of Medicine, Dentistry and Health Sciences, University of Melbourne, Melbourne, VIC, Australia; ${ }^{3}$ Central Clinical School, Faculty of Medicine, Nursing and Health Sciences, Monash University, Melbourne, VIC, Australia

\subsection{6/sextrans-2015-052270.549}

Introduction Studies of risk factors for HIV infection usually ascertain these at the time of diagnosis, when they are subject to multiple biases. This study aimed to identify factors present at the time of the last negative HIV test that could predict the risk of HIV acquisition within the next 12 months among men who have sex with men (MSM) attending a sexual health service in Australia. This may allow prioritisation of MSM for preventive interventions, which are becoming increasingly expensive.

Methods We conducted a retrospective cohort study of MSM attending Melbourne Sexual Health Centre between 1 January 2007 and 31 December 2013 with at least two HIV tests within 12 months. Age, sexual behaviour, and bacterial STI diagnoses were extracted from the date of the last negative HIV test and HIV incidence rate ratios (RR) were calculated for each risk factor. Risk factor prevalence was compared among all MSM and those subsequently infected.

Results Of 14745 MSM, 5262 were eligible, contributing 6525 person-years follow-up. 85 new HIV diagnoses were identified within 12 months of a HIV negative test with an incidence of 1.3 (95\% CI: 1.0-1.6) per 100 person-years. Significant associations with subsequent HIV infection were: gonorrhoea at any site (RR: 4.1, 95\% CI: 2.3-7.0), chlamydia RR: 3.9 (2.3-6.3), inconsistent condom use RR 2.7 (1.6-4.5), and injecting drugs RR 4.1 (1.7-8.6). Risk factor prevalences in tested MSM compared to those subsequently infected were: any bacterial STI ( $26 \%$ vs. $42 \%)$, inconsistent condom use (49\% vs. $73 \%)$, any significant risk factors (60\% vs. $83 \%)$.

Conclusion This analysis of a large clinic cohort identified significant predictors of subsequent HIV infection that were present at the last negative HIV test, when preventive intervention would still be possible. These could be used to prioritise MSM for potentially costly interventions.

Disclosure of interest statement We declare no conflict of interest.

\section{P16.03 COMPARISON OF SEXUAL RISK BEHAVIOURS AMONG HIV POSITIVE MEN WHO HAVE SEX WITH MEN BEFORE AND AFTER THEIR DIAGNOSIS}

${ }^{1}$ Gino M Calvo, 2,3Kelika A Konda, ${ }^{2}$ Segundo R León, ${ }^{2}$ Silver Vargas, 'Hugo Sánchez, ${ }^{4}$ Hector I Salvatierra, ${ }^{5}$ Brandon Brown, ${ }^{3}$ Jeffrey D Klausner, ${ }^{2}$ Carlos F Caceres. ${ }^{1}$ Epicentro, Lima, Peru; ${ }^{2}$ Unit of Health, Sexuality and Human Development, and Laboratory of Sexual Health, Universidad Peruana Cayetano Heredia, Lima, Peru; ${ }^{3}$ University of California, Los Angeles, CA, USA; ${ }^{4}$ Alberto Barton Health Center, Health Directorate of Callao, Lima, Peru; ${ }^{5}$ Program in Public Health, University of California, Irvine, CA, USA

10.1136/sextrans-2015-052270.550 